\title{
A Character Recognition Method for License Plate Based on Combination Feature and Partial Character Input
}

\author{
Qiang Li" ${ }^{1,}$, Juan Zhang ${ }^{2, b}$ \\ 1Shanghai University of Engineering and Technology, Shanghai, 201620, China; \\ ${ }^{2}$ Shanghai University of Engineering and Technology, Shanghai, 201620, China. \\ a953559760@qq.com, b793522121@qq.com
}

\begin{abstract}
In order to improve the recognition rate of license plate characters, a new method based on combination feature and partial character input is proposed. First, according to the texture characteristics of the license plate character, the basic LBP operator is improved, and then the characteristics of the license plate partial character image are extracted using the improved local binary mode and horizontal vertical projection, and the classifier is trained with the extracted features. Then use this classifier to classify and identify license plate characters. The experimental results show that the overall recognition rate of license plate reaches $94.56 \%$, which proves the effectiveness and robustness of this method.
\end{abstract}

Keywords: License plate character recognition, LBP feature, projection feature, BP neural network.

\section{Introduction}

License plate recognition is one of the important components in the intelligent transportation system. It is also a classic topic in the field of image recognition. It mainly involves three technologies: license plate positioning, character segmentation, and character recognition. Character recognition technology is the core and difficulty of the entire system[1], In this paper, an improved extraction method of LBP features and horizontal vertical projection features[2]-[4] is proposed. The characteristics of license plate characters are fully expressed using various features, and the method has a good adaptability to Chinese characters, letters and numbers.

\section{License Plate Character Recognition Feature Extraction}

\subsection{License Plate Character Recognition Preprocessing}

The preprocessing process [3]-[7] adopted in this paper is shown in Figure 1, and the preprocessed characters are then followed by feature extraction [8] and character recognition [9].

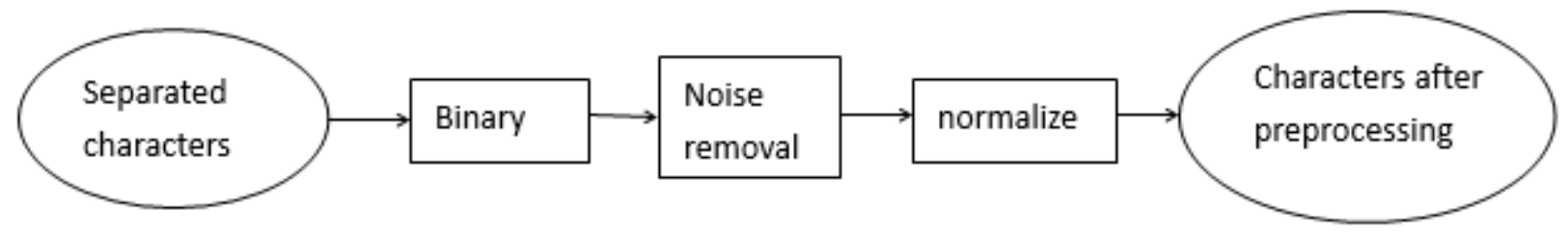

Fig. 1 Flow chart of pretreatment

\subsection{Character Feature Extraction}

\subsubsection{LBP Feature Extraction}

\section{(1) Improved LBP Operator}

On the basis of the basic LBP operator, according to the characteristics of the license plate characters, the basic LBP operator has been improved, and only two-thirds of the lower half of the 3 $\times 3$ pixel neighborhood is captured in the local binary mode descriptor. Therefore, there are 25 LBP eigenvalue modes for central pixels. While maintaining a high recognition rate, the speed of operation has also been greatly improved. Figure 2 is an example of an improved LBP operator. See formula(1). 


$$
L B P=\sum_{i=0}^{4} S\left(g_{i}-g_{c}\right) 2^{i}
$$

\begin{tabular}{|l|l|l|}
\hline 59 & 23 & 70 \\
\hline 42 & 50 & 88 \\
\hline 96 & 12 & 34 \\
\cline { 1 - 2 }
\end{tabular}

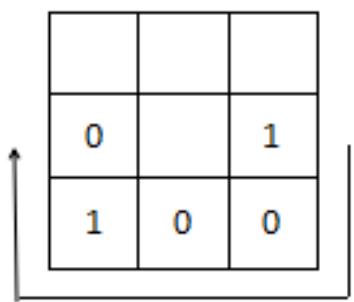

Binary number: 10010 decimal number:19

Fig. 2 Example of improved LBP operator base elements

\section{(2) LBP Statistical Histogram(LBPH)}

LBPH combines the LBP[10] feature with the spatial information of the image. This representation is presented by Ahonen et al. in the literature[ 16] In the proposal. The LBP feature image is divided into $\mathrm{N}$ rows $\mathrm{N}$ columns and $\mathrm{N}^{2}$ local blocks, and the histogram of each local block is extracted, and then these histograms are connected in turn to form a statistical histogram of $25 \times \mathrm{N}^{2}$ dimensional LBP feature vectors.

\subsubsection{Projection Feature Extraction}

For the normalized $\mathrm{M} \times \mathrm{M}$ character lattice[11], a horizontal projection is performed first, followed by a vertical projection, so that a total of $2 \times \mathrm{M}$ dimension projection feature vectors can be obtained. The specific process is shown in Figure 3.
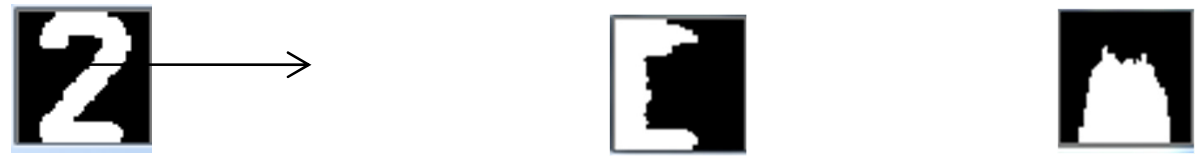

(a)Binary images (b) Horizontal projection histogram (c) Vertical projection histogram

Fig. 3 Projective feature extraction

\subsubsection{Part-character Features}

Compared with the method of full-character input, the partial character input method not only guarantees the effect of recognition but also improves the speed of recognition. At the same time, the partial character method also avoids the problem that adhesion characters are difficult to extract features[12]-[13]. If we select only $2 / 3$ of these 85 characters, we find that we can still recognize the characters and have a good degree of differentiation. Figure 4 shows the 16 characters and all letters and the partial characters of the numbers.

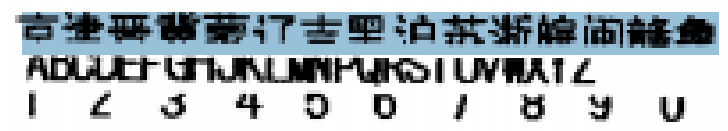

Fig. 4 The 16 characters and all letters and the partial characters of the numbers.

\section{License Plate Character Classification}

\subsection{BP Neural Network}

BP neural network [ 14] It is a typical multi-layer feedforward neural network with BP algorithm for error correction. It consists of two processes: the positive propagation of information and the reverse propagation of error. In this paper, a hidden layer of BP network is introduced, and multiple hidden layers are the same principle. In the BP neural network, a single sample has $\mathrm{M}$ inputs and $\mathrm{N}$ outputs. There are usually several hidden layers between the input layer and the output layer. In BP 
neural network, the number of nodes in input layer and output layer is determined, and the number of implicit nodes is uncertain. In fact, the number of implicit layer nodes has an impact on the performance of the neural network. There is an empirical formula that can determine the number of implicit layer nodes, see formula (2)

$$
h=\sqrt{m+n}+a
$$

Where $\mathrm{H}$ is the number of implicit layer nodes, $\mathrm{M}$ is the number of input layer nodes, $\mathrm{N}$ is the number of output layer nodes, and a is the adjustment constant between 1 and 10 . The design of neural network is very flexible. There is no absolute uniform standard. The structure and related parameters of the network are determined based on specific identified objects.

\subsection{Character Classification Process}

In this paper, we construct a sample set of feature vectors by feature extraction of license plate characters and use BP neural network[15] for classification study. The character classification process is shown in Figure 5.

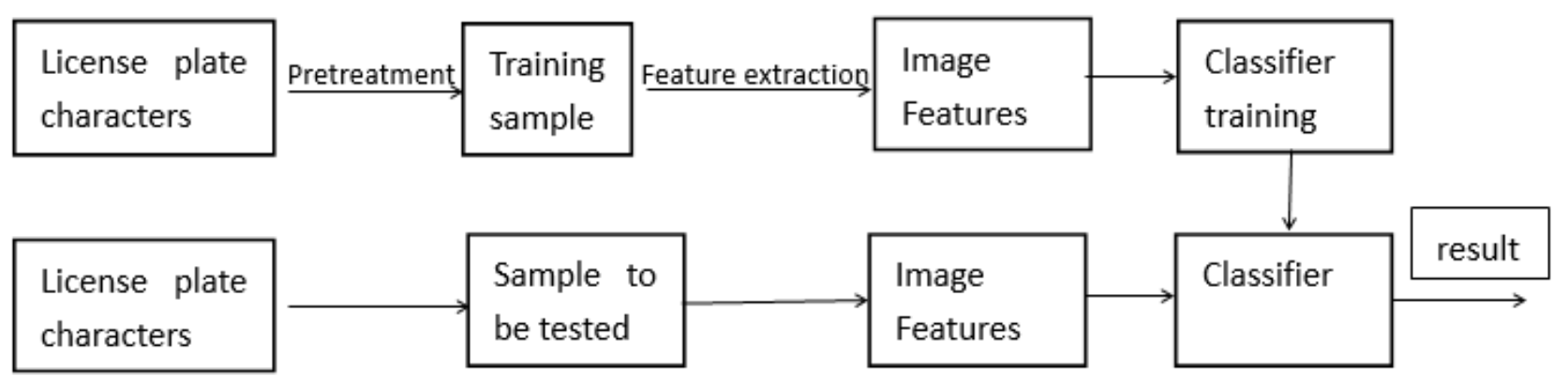

Fig. 5 Character Classification Flowchart

\section{Experimental Results and Analysis}

In this paper, $2 / 3$ of the license plate characters is normalized to $20 \times 20$ pixels. the horizontal vertical projection features of the character images are extracted to form a 40-dimensional feature vector. Secondly, the improved LBP feature extraction of the character image divides the extracted feature image into 4 rows and 4 columns with a total of 16 local blocks, extracts the histogram of each local block, forms a 512-dimensional LBP feature vector, and finally combines it. Can form 552 dimensional feature vectors. At the same time, it takes 552 neurons to set the BP neural network to the input layer, the number of implicit layer neurons is 30 , and the number of output neurons is 85 . The experimental results are shown in Figure 6 9.

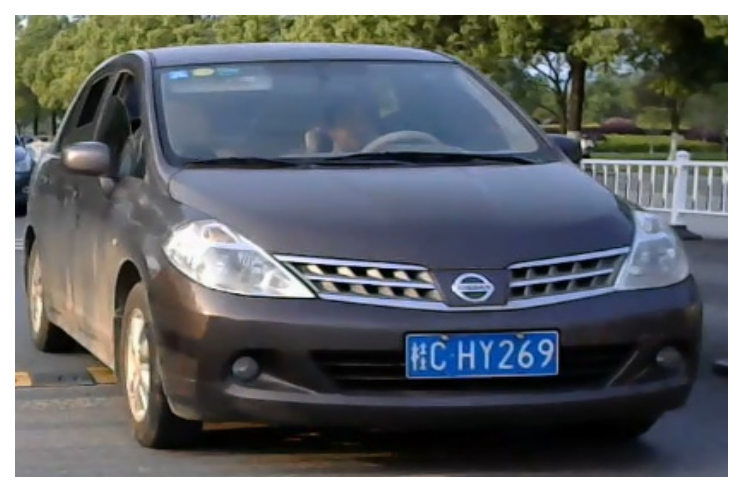

Fig. 6 Original 


\section{檗. HY269}

Fig. 7 Plates extracted

\section{HCI H I 269}

Fig. 8 Separated characters

\section{chars: 桂CHY269}

Fig. 9 License plate recognition results

The character image library used in this training and testing contains 13065 character images consisting of 51 Chinese characters, 24 letters(excluding I and O), and 10 numbers. On the same image test set, the proposed algorithm and other recognition algorithms are compared. The comparison results are shown in Table 1.

Table 1. Comparison of Recognition Results of Different Algorithm

\begin{tabular}{ccccc}
\hline & LBP & H+V & LBP+H+V & Modified LBP+ H+V \\
\hline Chinese character & $68.18 \%$ & $80.91 \%$ & $80.01 \%$ & $86.36 \%$ \\
Numbers, letters. & $80.91 \%$ & $96.36 \%$ & $92.73 \%$ & $96.36 \%$ \\
All Characters & $70.91 \%$ & $90.91 \%$ & $85.45 \%$ & $94.56 \%$ \\
\hline
\end{tabular}

\section{Conclusion}

In this paper, a method for identifying license plate characters based on combination features and BP neural network is proposed. In view of the shortcomings of single features, combined features are adopted: improved local binary mode(LBP) and horizontal vertical projection features. The feature extraction is carried out by using partial characters. Many features fully express the characteristics of the characters and have good adaptability to Chinese characters, numbers and letters. Experimental results show that the overall recognition rate reached $94.56 \%$. Through comparison with other license plate character recognition algorithms, the effectiveness and robustness of this method is demonstrated, and it has a certain practical value.

\section{References}

[1]. Hezhaocheng, Shexiwei, Yuwenjin, Yangwenchen. Character multifeature extraction method and its application in license plate recognition[ D] Computer Engineering and Application. 2011.47(23): 228-231.

[2]. Wangjue, Lihongyan.. Chinese plate character recognition algorithm based on local binary mode and multi-layer sensor [ A] Computer Applications, 2015,35(S1): 283-285, 304.

[3]. CHEN C-H, CHEN T-Y, WU M-T, et al. License plate recognition for moving vehicles using a moving camera [C]. Proceedings of the 2013 Ninth International Conference on Intelligent Information Hiding and Multimedia Signal Processing. Piscataway: IEEE, 2013:497 - 500.

[4]. Bulan O, Kozitsky V, Burry A M. Annotation free license plate recognition method and system: U.S. Patent 9,536,315[P]. 2017-1-3.

[5]. ZHOU W, LI H, LU Y, et al. Principal visual word discovery for automatic license plate detection [ J]. IEEE Transactions on Image Processing, 2012, 21( 9) : 4269 - 4279.

[6]. FAN X, FAN G. Graphical models for joint segmentation and recognition of license plate characters [ J]. IEEE Signal Processing Letters, 2009, 16( 1) : 10 - 13. 
[7]. Kim T, Kim E. A Vehicle License Plate Recognition System Using Morphological ROI (Region of Interest) Map Generated from Morphology Operation[C]//Journal of Physics: Conference Series. IOP Publishing, 2017, 806(1): 012004.

[8]. Raus M, Kreft L. Reading car license plates by the Use of artificial neural networks[J]. In: Proceedings of the 1995 IEEE 38th Midwest Symposium on Circuits and Systems, NJ, USA: IEEE 1995, Partl (of 2):538-541.

[9]. Parisi Reta1.Car Plate Recognition by Neural Networks and Image Processing[J]. Proceedings of the IEEE International Symposium on Circuits and Systems, 1998,195-198.

[10]. Zhangyin, Panyunhe. Pre-processing algorithm for vehicle license plate character recognition[J] . Computer Applications and Research, 1999.(7): 85:87.

[11]. OJALA T, HARWOOD D, PIETIKAINEN M. A comparative study introduction of texture measures with classification based on feature distributions [J] . Pattern Recognition, 1996, 29(7) : $51-59$.

[12]. AHONEN T, HADID A, PIETIKAINEN M. Face description with local binary patterns: application to face recognition [J]. IEEE Transactions on Pattern Analysis and Machine Intelligence, 2006,28( 12) : 2037 - 2041.

[13]. OJALA T, PIETIKAINEN M, MAENPAA T. Multi-resolution gray-scale and rotation invariant texture classification with local binary patterns [J]. IEEE Transactions on Pattern Analysis and Machine Intelligence, 2002, 24( 7) : 971 - 987.

[14]. Nielsen R H. Theory of the back propagation neural network. Proceeding of the International Joint Conference on Neural Networks,1989,1:593-605.

[15]. Wuyihong, Xugang, Jiangjuanjuan. An LBP-HOG feature fusion method for workpiece identification [J] Nanyang Normal University.

[16]. Leelawati Gaddam et al. License Plate Recognition.International Journal of Computer Science and Mobile Computing, Vol.3 Issue.9, September- 2014, pg. 492-495. 